\title{
Planejamento e implementação do curso Sexual Assault Nurse Examiner para o atendimento às vítimas de violência sexual: relato de experiência
}

\author{
Planning and implementation of the Sexual Assault Nurse Examiner \\ course to assist victims of sexual violence: an experience report \\ Planificación e implementación del curso Sexual Assault Nurse Examiner \\ para asistir a víctimas de violencia sexual: un informe de experiencia
}

Como citar este artigo

Silva JOM, Allen EM, Polonko I, Silva KB, Silva RC, Esteves RB. Planning and implementation of the Sexual Assault Nurse Examiner course to assist victims of sexual violence: an experience report. Rev Esc Enferm USP. 2021;55:e03739. https://doi.org/10.1590/S1980-220X2020029803739

\section{Juliana de Oliveira Musse Silva ${ }^{1}$ \\ Eileen Marie Allen² \\ Isle Polonko ${ }^{3}$ \\ Karen Beatriz Silva ${ }^{4}$ \\ Rita de Cassia Silva ${ }^{5}$ \\ Rafael Braga Esteves ${ }^{6}$}

${ }^{1}$ Hospital Universitário da Universidade Federal de Sergipe, Aracaju, SE, Brasil.

${ }^{2}$ Monmouth University, Nursing School, Monmouth, NJ, Estados Unidos da América.

${ }^{3}$ Clinical Practice Resources for

Training and Education, Hardwick,

NJ, Estados Unidos da América.

${ }^{4}$ Cedars-Sinai Medical Center,

Nursing Education, Los Angeles, CA,

Estados Unidos da América.

${ }^{5}$ Helping Hand of Los Angeles, Cedars-Sinai Medical Center, Los Angeles, CA, Estados Unidos da América.

${ }^{6}$ Universidade de São Paulo, Escola de Enfermagem de Ribeirão Preto, Departamento de Enfermagem Psiquiátrica e Ciências

Humanas, Ribeirão Preto, SP, Brasil.

\begin{abstract}
Objective: To describe the authors' experiences in planning and implementing a training course based on the Sexual Assault Nurse Examiner. Method: This is an experience report with qualitative characteristics of a descriptive nature and cross-sectional cut. In 2019 , nurses were trained to care for victims of sexual violence through a course known in the United States as Sexual Assault Nurse Examiner. The course lasted 40 hours and was offered to nurses. The didactic strategies used were dialogued exposure, group dynamics and realistic simulation for clinical-gynecological examination with volunteers. Results: For five days, 20 nurses underwent training. The contents covered Brazilian legislation, protocols for assisting victims, anamnesis, physical examination, collection and preservation of forensic remains. The practical classes took place in the last two days of training. At the end of the course, a written assessment was applied. Conclusion: The course provided the opportunity for the consolidation of important knowledge for nurses to care for victims of sexual violence, collection and preservation of traces and participation in the chain of custody.
\end{abstract}

\section{DESCRIPTORS}

Forensic Nursing; Education, Nursing; Violence Against Women; Violence; Nursing; Education.
Autor correspondente:

Rafael Braga Esteves

Rua João Ribeiro, 1454, Campos Elíseos

CEP 14085-700 - Ribeirão Preto, SP, Brasil rafael.braga.esteves@alumni.usp.br
Recebido: $16 / 07 / 2020$

Aprovado: 03/12/2020 


\section{INTRODUÇÃO}

A Organização Mundial da Saúde (OMS) explica violência sexual como atividades sexuais, tentativa para obter sexo, e insinuações sexuais, inadequadas ou não consentidas, com ou sem benefícios financeiros para os perpetradores ${ }^{(1)}$.

Neste contexto, mulheres estão entre os grupos vulneráveis a esse tipo de violência, tendo forte relação com as altas taxas de morbimortalidade para o sexo feminino nas diferentes faixas etárias e níveis socioeconômicos. No Brasil, entre os anos de 2017 e 2018, registraram-se 66.041 casos de violência sexual, sendo a maioria das vítimas do sexo feminino $(81,8 \%)$. Quanto ao perfil do agressor, verificou-se que $85,5 \%$ eram do sexo masculino e conhecido das vítimas ${ }^{(2)}$.

As consequências da violência sexual são diversas, quando física, as lesões genitoanais, como lacerações e fissuras, são recorrentes, bem como as doenças sexualmente transmissíveis $^{(3)}$. Além disso, consequências psicossociais estão presentes neste contexto, podendo ser ilustradas pelos transtornos psiquiátricos, dificuldades de aprendizagem, uso abusivo de drogas, perpetuação da violência e prostituição ${ }^{(4-5)}$.

Os serviços de saúde são espaços que atendem frequentemente as vítimas de violência sexual. Os enfermeiros, um dos integrantes das equipes de saúde mais presentes, possuem grande potencial para prestar um atendimento mais humanizado e resolutivo a esses pacientes ${ }^{(6)}$. Entretanto, a maior parte dos enfermeiros se sente despreparada para lidar com essas situações, seja pelo conhecimento limitado durante a anamnese e exame físico da vítima de violência sexual ou por não compreender a rede intersetorial de proteção às vítimas e o seu papel nesse contexto ${ }^{(7)}$.

Dessa forma, a assistência de enfermagem frente ao atendimento à vítima de violência sexual, na maioria das situações, é paliativa e restrita ao cuidado de lesões. Além disso, um dos grandes entraves para a visibilidade do problema é a falta de notificação pelos profissionais, o que colabora para o seu subdiagnóstico e subdimensionamento ${ }^{(8)}$.

Nos Estados Unidos, desde 1970, enfermeiros passaram a ser protagonistas no cuidado às vítimas de violência sexual. O Sexual Assault Nurse Examiner (SANE) surge como subespecialidade da enfermagem forense, no qual o profissional é habilitado a prestar cuidados à saúde de vítimas de agressão sexual, bem como na coleta e preservação de vestígios forenses, a fim de colaborar com as investigações criminais ${ }^{(9)}$.

Os enfermeiros habilitados como SANE recebem treinamento especializado para o reconhecimento de agressões sexuais, incluindo técnicas forenses para coleta de vestígios sem contaminação e preservação da cadeia de custódia, entrevista com os pacientes, identificação, avaliação e documentação de lesões ${ }^{(10)}$.

Diante do panorama exposto, e considerando a importância do processo de capacitação do enfermeiro para o atendimento integral às vítimas de violência sexual, o presente manuscrito objetivou descrever a experiência dos autores enfermeiros no planejamento e implementação de um curso de treinamento baseado no SANE.

\section{MÉTODO}

\section{Desenho do estudo}

Trata-se de um relato de experiência com características qualitativas de natureza descritiva, com recorte temporal transversal.

\section{Cenário}

O presente relato se refere a um curso de capacitação para enfermeiros brasileiros no atendimento às vítimas de violência sexual. A proposta do curso iniciou no mês de fevereiro de 2019, quando integrantes de uma organização sem fins lucrativos de enfermeiros forenses brasileiros contataram uma enfermeira forense responsável pela coordenação do programa de atendimento às vítimas de violência sexual de Nova Jersey, Estados Unidos da América (EUA), e uma instrutora de um programa de treinamento de "modelos vivos", o Forensic Gynecologic Teaching Associate (GTA), no intuito de propor a realização do SANE.

Para essa capacitação, foram realizadas 10 reuniões online com objetivo de organizar o curso, que aconteceu no mês de maio no ano de 2019. Na fase de planejamento, foi elaborado um cronograma com a sequência e distribuição dos conteúdos teóricos, abordagem didática e os materiais necessários para cada aula. O material didático escrito passou por um processo de tradução da língua inglesa para portuguesa por três tradutores.

O curso foi ministrado durante cinco dias consecutivos, com a presença de dois profissionais tradutores de inglês, nos períodos matutino e vespertino, o que totalizou a carga de 40 horas, divididas em 30 horas de aulas teóricas e 10 horas de aulas práticas. As estratégias didáticas utilizadas foram: exposição dialogada de conteúdo, dinâmicas em pequenos grupos, leitura e discussão de casos forenses. Para as aulas práticas, foi oportunizado o estudo aplicado em modelos humanos para a simulação realística.

Para a realização das simulações realísticas, 14 mulheres voluntárias foram modelos "vivas" e protagonizaram vítimas de violência durante as aulas práticas. Essas voluntárias eram alunas do curso de graduação em enfermagem de uma instituição privada, a qual uma das organizadoras do curso SANE esteve vinculada, e passaram por capacitação de 30 horas (quatro encontros online e quatro presenciais) para desenvolver a habilidade de atuar como usuárias durante a realização de todos os exames físicos e ginecológicos em seus corpos.

\section{Aspectos ÉtIcos}

O presente relato de experiência reflete as impressões dos autores deste texto sobre a vivência relatada e discutida nas seções deste artigo.

\section{RESULTADOS}

\section{A DESCRIÇÃO GERAL DO CURSO}

Durante cinco dias, 20 enfermeiros participaram do curso, tendo como instrutora uma enfermeira forense americana. 
Houve, também, a participação, durante as aulas teóricas, de uma enfermeira brasileira, responsável pela construção da rede de atendimento às vítimas de violência sexual do estado de Sergipe, uma advogada criminalista e uma delegada da Delegacia de Atendimento a Grupos Vulneráveis.

Os conteúdos teóricos abordados abrangeram: a história da enfermagem forense no mundo e sua inserção no Brasil; o papel do SANE nos EUA e suas práticas baseadas em evidências; recursos e materiais para a atuação do SANE; ética no atendimento às vítimas de violência; traumatologia forense; conhecimentos referentes a legislação brasileira; protocolos de atendimentos às vítima; o histórico (anamnese) e exame físico dos sobreviventes; coleta e preservação de vestígios forenses; avaliação e documentação de lesões.

No que concerne às aulas práticas, aconteceram nos dois últimos dias de treinamento nos laboratórios de enfermagem da universidade e abrangeram a realização do exame ginecológico em mulheres voluntárias durante a simulação realística do atendimento dos casos de violência sexual. Ao final do curso, uma avaliação escrita de aprendizagem, contendo 20 questões fechadas, foi aplicada aos 20 enfermeiros que participaram de todas as etapas do SANE, assim como uma avaliação de reação para avaliação da qualidade da capacitação.

\section{A ABORDAGEM TEÓRICA E PRÁTICA}

A organização dos conteúdos, durante as aulas teóricas, foi estabelecida buscando seguir uma sequência gradual e lógica de informações necessárias para o desenvolvimento de competências e habilidades dos enfermeiros durante o atendimento às vítimas de violência sexual (Quadro 1) e ocorreram durante os primeiros quatro dias de curso.

Quadro 1 - Blocos de conteúdos teóricos abordados no SANE Sergipe, Brasil, 2020.
1. Visão geral da enfermagem forense.
2. Papel do SANE nos EUA.
3. Noções de traumatologia forense.
4. Assistência de enfermagem no atendimento à vítima de violência sexual: anamnese/exame físico, documentação, registro e fotografia forense.
5. O papel do enfermeiro nos protocolos nacionais e internacionais.
6. Rede nacional e estadual de proteção às vítimas de violência sexual.

Fonte: Próprios autores.

O curso SANE é guiado por padrões e protocolos desenvolvidos por especialistas da área da Associação Internacional de Enfermeiras Forenses (International Association of Forensic Nurses - IAFN), consolidados por meio da publicação do guia intitulado Diretrizes de Educação para Enfermeiros Examinadores de Assalto Sexual. Os componentes dessas diretrizes servem para: definir a população de pacientes; estabelecer a duração da instrução; incluir recomendações para estudantes e educadores; estabelecer uma descrição detalhada do conteúdo e especificar recomendações para a preceptoria clínica ${ }^{(10)}$.

Por meio de várias discussões, os planejadores do programa foram capazes de criar conteúdo que atendia ou excedia os requisitos mínimos das diretrizes identificadas, mas também adaptado para atender às necessidades exclusivas do público de enfermeiros pioneiros que participam desse programa inaugural. Desde os estágios iniciais do planejamento, ficou claro que os enfermeiros estavam dispostos a superar quaisquer obstáculos encontrados. Os participantes demonstraram entusiasmo com a aproximação dos novos conceitos e habilidades apresentadas e a expectativa de inovar o atendimento nas próprias comunidades. Os materiais do curso foram preparados e distribuídos com antecedência, permitindo que os participantes se familiarizassem com os conteúdos teórico-científicos que compõem o curso SANE.

Com base no entendimento da variedade de estilos bem-sucedidos de aprendizagem de adultos, a agenda do curso de instrução foi apresentada em uma variedade de metodologias. Incluiu palestra verbal, apresentação de slides e vídeo, demonstração visual, discussão em grupo e projetos em grupo. As 30 horas de conteúdo (Quadro 1) em sala de aula integraram teoria e pesquisa, com demonstrações de habilidades clínicas. $\mathrm{Na}$ sala de aula, foi dada grande ênfase à educação dos enfermeiros sobre os aspectos exclusivos do trabalho com paciente com traumas sugestivos de agressões sexuais.

A importância de esclarecer a vítima quanto à abordagem centrada no trauma informado para comunicação, e a avaliação física foi explicada e discutida detalhadamente com os alunos. Foi apresentada a introdução de conceitos associados à neurobiologia do trauma, juntamente com uma revisão da psicologia da vitimização. Especialistas locais e regionais das comunidades policiais e legais instruíram sobre o modo como o sistema jurídico opera nesses casos, enquanto que os instrutores de enfermagem se concentraram no que essas enfermeiras poderiam fazer para auxiliar o processo de investigação, mantendo o foco na vítima. Foram ensinadas e demonstradas habilidades e técnicas específicas para coletar espécimes legalmente admissíveis, fotografar feridas e documentar minunciosamente sinais e sintomas.

As atividades práticas foram realizadas nos dois últimos dias de treinamento. No primeiro dia de prática, a instrutora realizou a demonstração em sala de aula do uso dos materiais necessários durante o atendimento às vítimas de violência sexual, bem como de um kit utilizado nos EUA para a coleta dos vestígios colhidos e a correta preservação da cadeia de custódia. Nesse momento, os enfermeiros puderam tocar e manejar alguns materiais, tais como swabs e espéculos. A seguir, a instrutora dividiu os enfermeiros em 4 grupos para a demonstração prática completa do exame em uma modelo viva no laboratório.

O espaço de laboratório, fornecido por uma instituição de ensino superior privada, foi dividido em seções por cortinas e divisórias para criar quatro salas de exame simuladas diferentes, e cada uma das oito diferentes GTA forenses participaram de duas sessões de exames físicos consecutivas. Cada grupo de enfermeiros do programa de treinamento da SANE se reunia com uma GTA responsável por passar a técnica correta para realização do exame ginecológico. 
Foram fornecidas, aproximadamente, 10 horas de aprendizado visual e experimental com as GTA por meio de demonstração instrucional de inspeção genital forense, fotografia forense e coleta de evidências. Os alunos tiveram a oportunidade de demonstrar as habilidades técnicas e de comunicação verbal e não verbal adequada ao atendimento às vítimas.

O último dia do treinamento do SANE consistiu na simulação do atendimento de enfermagem pelos profissionais, com a participação das GTA que faziam o papel de sobreviventes de violência sexual. Nesse momento, os enfermeiros puderam pôr em prática os conhecimentos necessários para a realização das entrevistas e o uso do kit de coleta de evidências forenses.

Cada grupo se dirigiu a um ambiente de simulação e atribuições foram delegadas para cada membro. Um enfermeiro ficava responsável por realizar a anamnese, outro, pelo exame físico, um, pela coleta de vestígios e outro observava todos os procedimentos para realizar o feedback, ao final, com os demais. Apesar das divisões de tarefas, todos os enfermeiros fizeram a prática de exame ginecológico nas voluntárias e esse processo foi supervisionado por uma das instrutoras, responsável pela aula prática.

$\mathrm{Na}$ conclusão da agenda do curso, cada aluno era obrigado a responder um exame escrito para avaliar o conhecimento adquirido. Um foco particular foi colocado na capacidade do enfermeiro de avaliar criticamente os detalhes exclusivos que eles poderiam encontrar e desenvolver um plano de atendimento personalizado para o paciente. Todos os alunos do curso inaugural finalizaram com êxito a avaliação.

\section{DISCUSSÃO}

O enfermeiro SANE, com as habilidades para entrevista forense, exame físico e coleta de vestígios, é a intersecção entre a saúde e justiça, utilizando de forma objetiva partes dos conceitos que compõem a especialidade enfermagem forense ${ }^{(11)}$. Estudos presentes na literatura mostram a eficácia dos programas SANE em muitos aspectos, como na recuperação psicológica dos pacientes, na instituição do tratamento precoce (contracepção de emergência, profilaxia para infecções sexualmente transmissíveis), bem como no registro completo e preciso de vestígios forenses ${ }^{(10-12)}$.

Nos EUA, os exames de agressão sexual concluídos pelas SANE podem colaborar no processo de apuração da absolvição ou condenação de um suspeito devido à melhor coleta de vestígios. Para a obtenção de resultados como os descritos anteriormente, é necessário que esses profissionais passem por um processo de treinamento teórico-prático com abordagem didática efetiva e condicente com os protocolos estaduais e nacionais ${ }^{(13)}$.

Esse treinamento normalmente inclui uma carga horária de 40 horas entre conteúdos teórico-científicos e a pratica de exame ginecológico simulado. Ainda pode incluir a realização de três exames em vítimas de agressão sexual, acompanhados por preceptor SANE experiente em campo ${ }^{(10)}$.

Vale ressaltar que o aprendizado é um processo no qual o conhecimento é apreendido também através da experiência prática. Nesse sentido, a simulação realística é uma metodologia que permite que o educando aplique a teoria abordada em sala de aula com maior desenvoltura na prática e de forma segura ${ }^{(14)}$.

Métodos tradicionais de ensino pautados na simulação realística utilizando robôs, cadáveres, ou até mesmo atores sem treinamento, possuem mais limitações, quando comparado ao ensino pratico em "modelos vivos", como o exemplo do programa de treinamento com a sigla em inglês GTA. Nesse programa, as "modelos vivas" são capacitadas para se comunicar e instruir os alunos através do feedback em tempo real quanto às técnicas de exame de habilidades clínicas invasivas ${ }^{(15)}$.

No Brasil, o enfermeiro participa do atendimento de vítimas de violência sexual, sobretudo em serviços de referência, mas suas ações ainda são centradas no tratamento e não na preservação de vestígios forenses e sua participação na cadeia de custódia ainda é questionada. Embora o Decreto $\mathrm{n}^{\circ} 7.958 / 2013^{(16)}$ e a Portaria $\mathrm{n}^{\circ} 288 / 2015^{(17)}$ contemplem a preservação e coleta de vestígio como etapa do atendimento integral no serviço de saúde, esses procedimentos são apontados como atividades médicas, com a necessária presença de outro profissional de saúde pela Norma Técnica de $2015^{(18)}$, criada para esse fim.

Contudo, novas perspectivas de aprimoramento profissional para o atendimento às vítimas de violência sexual surgem no país com o reconhecimento da especialidade enfermagem forense pelo Conselho Federal de Enfermagem (Cofen), em 2011, pela Resolução $\mathrm{n}^{\circ} 389^{(19)}$ e a regulamentação das áreas de atuação do enfermeiro forense em 2017, através da Resolução n $556^{(20)}$.

\section{CONCLUSÃO}

No Brasil, o curso SANE foi realizado pela primeira vez no período de 6 a 8 de maio de 2019, como relatado neste artigo, sendo uma iniciativa de treinamento de capacitação de alta performance para enfermeiros assistirem vítimas de violência sexual. Os objetivos contemplados no curso convergem com as diretrizes preconizadas pela legislação brasileira para o atendimento integral às vítimas de violência, como a participação do profissional de saúde na preservação e coleta dos vestígios forenses, bem como a garantia da idoneidade da cadeia de custódia nos serviços de saúde. Este relato de experiência é importante, pois sintetiza uma experiência brasileira que contou com profissionais de ampla experiência na formação de enfermeiros SANE dos EUA, além de ter sido composto por enfermeiros com amplo conhecimento na área.

Objetivo: Descrever a experiência dos autores enfermeiros no planejamento e implementação de um curso de treinamento baseado no Sexual Assault Nurse Examiner. Método: Trata-se de um relato de experiência com características qualitativas de natureza descritiva e recorte transversal. No ano de 2019, foram treinados enfermeiros para o atendimento de vítimas de violência sexual por meio de um curso, nos Estados Unidos, conhecido como Sexual Assault Nurse Examiner. O curso teve carga horária de 40 horas e foi oferecido para 
enfermeiros. As estratégias didáticas utilizadas foram: exposição dialogada, dinâmicas em grupos e simulação realística para o exame clínico-ginecológico com voluntárias. Resultados: Durante cinco dias, 20 enfermeiros foram capacitados. Os conteúdos abordados abrangeram a legislação brasileira, protocolos de atendimentos às vítimas, a anamnese, exame físico, coleta e preservação de vestígios forenses. As aulas práticas aconteceram nos dois últimos dias de treinamento. Ao final do curso, aplicou-se uma avaliação escrita. Conclusão: $\mathrm{O}$ curso oportunizou a consolidação de conhecimentos importantes para o atendimento pelo enfermeiro às vítimas de violência sexual, coleta e preservação de vestígios e participação na cadeia de custódia.

\section{DESCRITORES}

Enfermagem Forense; Educação em Enfermagem; Violência contra a Mulher; Violência; Enfermagem; Educação.

\section{RESUMEN}

Objetivo: Describir la experiencia de enfermeras autoras en la planificación e implementación de un curso de capacitación basado en el Sexual Assault Nurse Examiner. Método: Se trata de un relato de experiencia con características cualitativas de carácter descriptivo y de corte transversal. En 2019, se capacitó a enfermeras para atender a víctimas de violencia sexual a través de un curso en los Estados Unidos, conocido como Sexual Assault Nurse Examiner. El curso tuvo una carga de trabajo de 40 horas y se ofreció a enfermeras. Las estrategias didácticas utilizadas fueron: exposición de diálogo, dinámica de grupo y simulación realista para examen clínico-ginecológico con voluntarios. Resultados: Durante cinco días se capacitó a 20 enfermeras. Los contenidos cubrieron la legislación brasileña, protocolos de atención a las víctimas, anamnesis, exploración física, recolección y preservación de restos forenses. Las clases prácticas tuvieron lugar en los dos últimos días de formación. Al finalizar el curso se aplicó una evaluación escrita. Conclusión: El curso brindó una oportunidad para la consolidación de conocimientos importantes para los enfermeros en la atención de víctimas de violencia sexual, recolección y preservación de huellas y participación en la cadena de custodia.

\section{DESCRIPTORES}

Enfermería Forense; Educación en Enfermería; Violencia contra la Mujer; Violencia; Enfermería; Educación.

\section{REFERÊNCIAS}

1. Krug EG, Dahlberg LL, Mercy JA, Zwi AB, Lozano R. World report on violence and health [Internet]. Geneva: WHO; 2002 [cited 2020 Jan 11]. Available from: https://apps.who.int/iris/bitstream/handle/10665/42495/9241545615_eng.pdf;jsessionid=B843D46865D80159E 8E8BB03E34FD4BF?sequence=1

2. Fórum Brasileiro de Segurança Pública. Anuário brasileiro de segurança pública 2019 [Internet]. São Paulo: Fórum Brasileiro de Segurança Pública; 2019 [cited 2020 Jun 27]. Available from: https://www.forumseguranca.org.br/wp-content/uploads/2019/10/Anuario-2019FINAL_21.10.19.pdf

3. Avanci JQ, Pinto LW, Assis SG. Treatment for cases of violence by Brazilian emergency services focusing on family relationships and life cycles. Cienc Saúde Coletiva. 2017;22(9):2825-40. https://doi.org/10.1590/1413-81232017229.13352017

4. Platt VB, Back IC, Hauschild DB, Guedert JM. Sexual violence against children: authors, victims and consequences. Cienc Saúde Coletiva. 2018;23(4):1019-31. https://doi.org/10.1590/1413-81232018234.11362016

5. Delziovo CR, Coelho EB, D'orsi E, Lindner SR. Sexual violence against women and care in the health sector in Santa Catarina-Brazil. Cienc Saúde Coletiva. 2018;23(5):1687-96. https://doi.org/10.1590/1413-81232018235.20112016

6. Martins DC, Gois OJ, Silva JO, Rosa MPS, Gonçalves MC. Violência: abordagem, atuação e educação em enfermagem. Cienc Biolog Saúde Unit [Internet]. 2017 [cited 2020 Jun 27];4(2):155-68. Available from: https://periodicos.set.edu.br/index.php/cadernobiologicas/ article/view/4603

7. Souza AC, Marques CS, Souza Neto CM, Martins IS, Musse JO, Gonçalves M. [The nurse and the preservation of vestiges against sexual violence against women]. Nursing (São Paulo). 2017;20(233):1878-82. Portuguese.

8. Garbin CA, Dias IA, Rovida TA, Garbin AJ. [Challenges facing health professionals in the notification of violence: mandatory implementation and follow-up procedures]. Cienc Saúde Coletiva. 2015;20(6):1879-90. Portuguese. https://doi.org/10.1590/141381232015206.13442014

9. Morse J. Legal mobilization in medicine: Nurses, rape kits, and the emergence of forensic nursing in the United States since the 1970s. Soc Sci Med. 2019 Feb;222:323-34. https://doi.org/10.1016/j.socscimed.2018.12.032

10. International Association of Forensic Nurses. Sexual Assault Nurse Examiner (SANE): education guidelines [Internet]. Harrisburg: International Association of Forensic Nurses; 2018 [cited 2020 Jun. 27]. Available from: https://cdn.ymaws.com/www.forensicnurses.org/ resource/resmgr/education/2018_sane_edguidelines.pdf

11. Conselho Federal de Enfermagem. Resolução Cofen $N^{\circ}$ 0556, de 23 de agosto de 2017. Regulamenta a atividade do Enfermeiro Forense no Brasil, e dá outras providências [Internet]. Brasília, DF: COFEN; 2017 [cited 2020 Jun 27]. Available from: http://www.cofen.gov.br/ resolucao-cofen-no-05562017_54582.html

12. Thomas TL, Nobrega JC, Britton-Susino S. Rural health, forensic science and justice: a perspective of planning and implementation of a sexual assault nurse examiner training program to support victims of sexual assault in rural underserved areas. Forensic Sci Int: Reports. 2020;2:100053. https://doi.org/10.1016/j.fsir.2019.100053

13. Marks SA, Kaiser L, Mccleery MB. A novel approach to sexual assault nurse examiner training: a pilot program. Clin Simul Nurs. 2017;13(12):595-600. https://doi.org/10.1016/j.ecns.2017.07.002

14. Damewood AM. Current trends in higher education technology: simulation. TechTrends. 2016;60(3):268-71. https://doi.org/10.1007/ s11528-016-0048-1

15. Association of American Medical Colleges. AAMC statement on patient rights and medical training [Internet]. Washington, DC: AAMC; 2003 [cited 2020 Jun 27]. Available from: https://web.archive.org/web/20030623113147/ https://www.aamc.org/newsroom/ pressrel/2003/030612.htm 2003 
16. Brasil. Decreto $N^{\circ} 7.958$, de 13 de março de 2013. Estabelece diretrizes para o atendimento às vítimas de violência sexual pelos profissionais de segurança pública e da rede de atendimento do Sistema Único de Saúde. Diário Oficial da União, Brasília, DF, 2013 Mar 14. Section 1, p. 1.

17. Brasil. Ministério da Saúde. Portaria № 288, de 25 de março de 2015. Estabelece orientações para a organização e integração do atendimento às vítimas de violência sexual pelos profissionais de segurança pública e pelos profissionais de saúde do SUS. Diário Oficial da União, Brasília, DF; 2015 Mar 26.

18. Brasil. Ministérios da Saúde, Ministério da Justiça, Secretaria de Políticas para as Mulheres. Norma técnica: Atenção humanizada às pessoas em situação de violência sexual com registro de informações e coletas de vestígios. Brasília, DF: Secretaria de Polítcas para as Mulheres; 2015

19. Conselho Federal de Enfermagem. Resolução Cofen № 389, 18 de outubro de 2011. Atualiza, no âmbito do Sistema Cofen /Conselhos Regionais de Enfermagem, os procedimentos para registro de título de pós-graduação lato e stricto sensu concedido a enfermeiros e lista as especialidades [Internet]. Brasília, DF: COFEN; 2011 [cited 2020 Jun 27]. Available from: http://www.cofen.gov.br/resoluocofen-n-3892011_8036.html

20. Conselho Federal de Enfermagem. Resolução Cofen $N^{\circ}$ 556, 18 de outubro de 2017. Regulamenta a atividade do Enfermeiro Forense no Brasil, e dá outras providências [Internet]. Brasília, DF: COFEN; 2017 [cited 2020 Jun 27]. Available from: http://www.cofen.gov.br/wpcontent/uploads/2017/08/RES.-556-2017-PUBLICA\%C3\%87\%C3\%83O-NO-D.O.U..pdf 